\title{
Is a glowing LED meaningful to determine the Plank's constant accurately?
}

\section{Chetan Kotabage}

Chetan Kotabage, "Is a glowing LED meaningful to determine the Plank's constant accurately?," Proc. SPIE 11143, Fifteenth Conference on Education and Training in Optics and Photonics: ETOP 2019, 111432S (2 July 2019); doi: $10.1117 / 12.2508193$ 


\title{
Is a glowing LED meaningful to determine Planks constant accurately?
}

\author{
Chetan Kotabage \\ Department of Physics, KLS Gogte Institute of Technology, Belagavi, India
}

\begin{abstract}
Plank's constant is a fundamental constant in Physical sciences and Millikan was awarded Noble prize for its experimental determination. With the advent of solid state electronic devices, experiments were designed to determine the Plank's constant utilizing LED. ${ }^{1,2}$ Herrmann, Schätzle ${ }^{3}$ and Morehouse $^{4}$ have discussed the validity of this experiment. In this paper, the validity of this experiment is re-examined. The measurements of the longest wavelength light emitted by LED and precise determination of band gap energy of LED are essential for accurate determination of Plank's constant by utilizing LED. ${ }^{5}$ An experimental study of these arguments is also presented in this paper.
\end{abstract}

Keywords: Plank's constant, LED

\section{INTRODUCTION}

Plank's constant was initially determined experimentally by photoelectric effect. In photoelectric effect, absorption of a photon of appropriate energy by a metal results in ejection of an electron. If energy of the photon is larger than work function of metal, kinetic energy of these photoelectrons is non-zero. The maximum kinetic energy of photoelectrons can be estimated over a range of energy of photons. To this end, measurement of stopping potential as a function of frequencies of radiation is carried out. These measurements yield Plank's constant experimentally. ${ }^{6}$

In the working of light emitting diode (LED), a somewhat reverse mechanism is observed. This mechanism has been utilized to determine Plank's constant. In Sec. 2, determination of Plank's constant as described in Ref. 1,2 is discussed. The drawbacks of this technique and modifications to obtain more accurate result are discussed in Sec. 3. In Sec. 4, experimental results and discussion about these results is presented.

\section{DETERMINATION OF PLANK'S CONSTANT USING LED}

The light emission in LED results from recombination of electrons in the conduction band and holes in the valence band. The minimum energy of a photon, which has been emitted in this process, is equal to the band gap energy, $E_{g}$. If the wavelength, $\lambda$, of radiation corresponding to this photon is known precisely, then a measurement of band gap energy can yield Plank's constant, $h$, as

$$
h=\frac{\lambda E_{g}}{c},
$$

where $c$ is speed of light. The use of LED to find Plank's constant is based on this equation. As described in Ref. 1,2, I-V characteristics of LED are measured to estimate the band gap energy. The intercept of linear part of I-V characteristics curve on voltage axis, which called as forward voltage $V_{F}$, is assumed to be equal to $E_{g} / q^{*}$, where $q$ is the charge of electron.

Thus, from Eq. (1), Plank's constant is given as

$$
h=\frac{\lambda V_{F} q}{c} .
$$

Further author information: E-mail: cvkotabage@git.edu, Telephone: 918312498500

${ }^{*}$ It is mentioned in Ref. 1 that forward voltage is about $90 \%$ of $E_{g}$. 


\section{DRAWBACKS OF THE METHOD}

The experimental determination of Plank's constant by above method has the following drawbacks.

\subsection{Wavelength of light}

For accurate calculation of Plank's constant using Eq. (2), the longest wavelength of light should be considered. The wavelength of light depends on energy difference between a level of conduction band and a level of valence band, which are involved in the transition of electron from conduction band to valence band. The band gap energy, $E_{g}$, has lowest energy difference because of energy difference between the lowest level of conduction band and the highest level of valence band. Thus, apart from photons of energy $E_{g}$, all other photons emitted by LED will be of higher energy than $E_{g}$. Since the wavelength of light is inversely proportional to energy of corresponding photon

$$
E=\frac{h c}{\lambda},
$$

a photon of energy $E_{g}$ will correspond to the longest wavelength of light. Since Eq. (2) is obtained for photon of energy $E_{g}$, the longest wavelength of light must be considered.

Usually, the calculations involve the wavelength specified by the manufacturer, which is not essentially the longest wavelength emitted by LED. Thus, incorrect wavelength of light in Eq. (2) results in inaccurate Plank's constant.

\subsection{Forward voltage}

The forward voltage is determined by the intercept of linear part of I-V characteristics on the voltage axis. This technique of defining band gap energy by utilizing forward voltage is not correct as the intercept is not equal to $E_{g} / q$. At forward voltage, diffusion of electrons and holes across the junction begins due to decrease in junction potential. At this voltage, respective conduction and valence bands of $n$ and $p$ type semiconductors shift closer. Hence, the product of charge of electron and forward voltage of a diode is less than band gap energy.

As mentioned in Ref. 7, the product of limiting forward voltage of a diode and charge of electron is slightly less than the band gap energy. At limiting forward voltage, the highest possible shift between respective conduction and valence bands of $\mathrm{n}$ and $\mathrm{p}$ type semiconductors takes place. Beyond the limiting forward voltage, the diode equation,

$$
I=I_{0}\left(e^{\eta e V / K T}-1\right),
$$

does not hold for LED. This argument can be confirmed by plotting a graph of $\ln I$ vs voltage. After limiting forward voltage, $\ln I$ vs. voltage curve deviates from its linear nature.

Hence, instead of forward voltage, utilization of limiting forward voltage will result in more accurate estimation of Plank's constant.

\section{EXPERIMENT AND DISCUSSION}

The experimental set up to determine Plank's constant using Blue, Green, Yellow and Red LEDs is shown in Fig. 1. I-V characteristics of each LED are shown in Fig. 2. As discussed in Sec. 3.2, forward voltage can be estimated by extending the linear part of this graph and finding the intercept on the voltage axis. Fig. 2 shows estimation of forward voltage for each LED.

In Eq. (4), the factor, $e^{\eta e V / K T}$, is much greater than 1 at room temperature. Hence, natural logarithm of Eq. (4) yields

$$
\ln I=\frac{\eta e V}{K T}+\ln I_{0},
$$

which indicates that graph of $\ln I$ vs. voltage is a straight line as long as LED follows diode equation. In Fig. 3, the graph of $\ln I$ vs. voltage is shown for each LED. The limiting forward voltage for each LED is defined as the voltage beyond which this curve deviates from its linear nature.

The spectrum in Fig. 4 of each LED was recorded using Ocean Optics HR4000 spectrometer. The wavelength, where maximum intensity is observed, is not considered as the maximum wavelength of radiation emitted by LED. 
To estimate the maximum wavelength, spectral width is measured for each spectrum. The longest wavelength at full width at half maximum is considered as the maximum wavelength emitted by LED. This method is opted because the spectrum of each LED is spread over a range of about 60 to $80 \mathrm{~nm}$. As argued in Sec. 3.1, energy of photon corresponding to radiation at the maximum wavelength is equal to band gap energy. Table 1 lists these variables for each LED.

Table 1. Forward voltage and limiting forward voltage, peak wavelength and maximum wavelength measurements for Blue, Green, Yellow and Red LEDs

\begin{tabular}{|l|l|l|l|l|}
\hline Colour & $\begin{array}{l}\text { Forward voltage } \\
(\mathrm{V})\end{array}$ & $\begin{array}{l}\text { Limiting forward } \\
\text { voltage }(\mathrm{V})\end{array}$ & $\begin{array}{l}\text { Peak } \\
\text { wavelength }(\mathrm{nm})\end{array}$ & $\begin{array}{l}\text { Maximum } \\
\text { wavelength }(\mathrm{nm})\end{array}$ \\
\hline Blue & 2.62 & 2.66 & 466.51 & 483.67 \\
\hline Green & 1.86 & 1.93 & 567.07 & 586.03 \\
\hline Yellow & 1.85 & 1.88 & 594.22 & 604.18 \\
\hline Red & 1.92 & 1.96 & 626.86 & 636.01 \\
\hline
\end{tabular}

Tables 2, 3, 4 list Plank's constant calculated from Eq. (2). The percentage error is calculated for the accepted value of $6.626 \times 10^{-34}$ Js. In Table 2 , forward voltage and peak wavelength, in Table 3, limiting forward voltage and peak wavelength, and in Table 4, limiting forward voltage and maximum wavelength were used for calculation.

Table 2. Estimated Plank's constant using forward voltage and peak wavelength.

\begin{tabular}{|l|l|l|}
\hline Colour & Plank's constant $\left(\times 10^{-34} \mathrm{Js}\right)$ & Percentage error $\%$ \\
\hline Blue & 6.53 & 1.45 \\
\hline Green & 5.64 & 14.9 \\
\hline Yellow & 5.87 & 11.4 \\
\hline Red & 6.43 & 2.96 \\
\hline
\end{tabular}

Table 3. Estimated Plank's constant using limiting forward voltage and peak wavelength.

\begin{tabular}{|l|l|l|}
\hline Colour & Plank's constant $\left(\times 10^{-34} \mathrm{Js}\right)$ & Percentage error $\%$ \\
\hline Blue & 6.63 & 0.0604 \\
\hline Green & 5.85 & 11.7 \\
\hline Yellow & 5.97 & 9.90 \\
\hline Red & 6.57 & 0.845 \\
\hline
\end{tabular}

From the comparison of percentage error in Table 2 and 4, the method of using limiting forward voltage and maximum wavelength yields more accuracy. The percentage error in Table 3 indicates that the results obtained by using peak wavelength is significantly less only for blue LED. The higher percentage error for blue LED in Table 4 is because of larger value of maximum wavelength. This deviation is due to inability to determine wavelength of radiation corresponding to a photon emitted by an electron in transition from the lowest level of conduction band and the highest level of valence band correctly. Currently, there is no method available to determine it experimentally. For direct or indirect band gap materials that are used in LEDs, energy levels below the lowest level of conduction band are introduced to achieve efficient emission of radiation. It causes radiation at higher wavelengths in the spectrum than the desired wavelength. The results of blue LED in Table 3 and 4 indicate that it is difficult to identify the desired wavelength in spectrum. 
Table 4. Estimated Plank's constant using limiting forward voltage and maximum wavelength.

\begin{tabular}{|l|l|l|}
\hline Colour & Plank's constant $\left(\times 10^{-34} \mathrm{Js}\right)$ & Percentage error $\%$ \\
\hline Blue & 6.87 & 3.68 \\
\hline Green & 6.04 & 8.84 \\
\hline Yellow & 6.07 & 8.39 \\
\hline Red & 6.66 & 0.513 \\
\hline
\end{tabular}

\section{CONCLUSIONS}

For accurate determination of Plank's constant, exact measurement of band gap energy of LED and wavelength of radiation caused by a transition from lowest level of conduction band and highest level of valence band are essential. Though use of LED to determine Plank's constant gives values that are close to Plank's constant, the experimental procedure described in Ref. 1,2 needs modification to obtain more accurate results. As suggested in Ref. 5, more accurate results can be obtained, if longest wavelength of radiation and limiting forward voltage are used for estimation. The experimental results confirm it except for blue LED.

This exception indicates that exact determination of desired wavelength of radiation in Eq. 1 remains elusive. Besides, band gap energy determination from limiting forward voltage is also not exact. Hence, though the modifications yield better results, Plank's constant cannot be determined accurately by using LED.

\section{ACKNOWLEDGMENT}

I thank Dr. Sushil Mujumdar and Mr. Sandip Mondal, Nano-optics and Mesoscopic Optics Laboratory, Department of Nuclear and Atomic Physics, Tata Institute of Fundamental Research, Mumbai for spectroscopic measurements. I thank anonymous referee of Resonance-Journal of Science Education.

\section{REFERENCES}

[1] O'conner, P. J. and O'conner, L. R., "Measuring plank's constant using a light emitting diode," The Physics Teacher 12, 423-425 (1974).

[2] Nieves, L., Spavieri, G., Fernandez, B., and Guevara, R. A., "Measuring the plank's constant with led's," The Physics Teacher 35, 108-109 (1997).

[3] Herrmann, F. and Schätzle, D., "Question \#53. measuring plank's constant by means of an led," Am. J. Phys. 64 (12), 1418 (1996).

[4] Morehouse, R., "Answer to question \#53. measuring plank's constant by means of an led," Am. J. Phys. 66, 12 (1998).

[5] Kotabage, C., "Is a glowing led useful for accurate determination of plank's constant?," arXiv:1811.05799 [physics.ed-ph] (2018).

[6] Krane, K., [Modern Physics], John Wiley \& sons Pvt. Ltd, Singapore (1996).

[7] Streetman, B. G. and Banerjee, S., [Solid state electronic devices], Prentice Hall of India Pvt. Ltd, New Delhi (2011). 


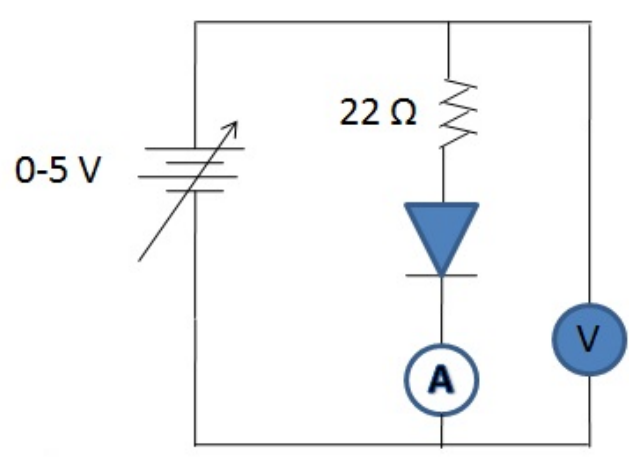

Figure 1. Circuit diagram

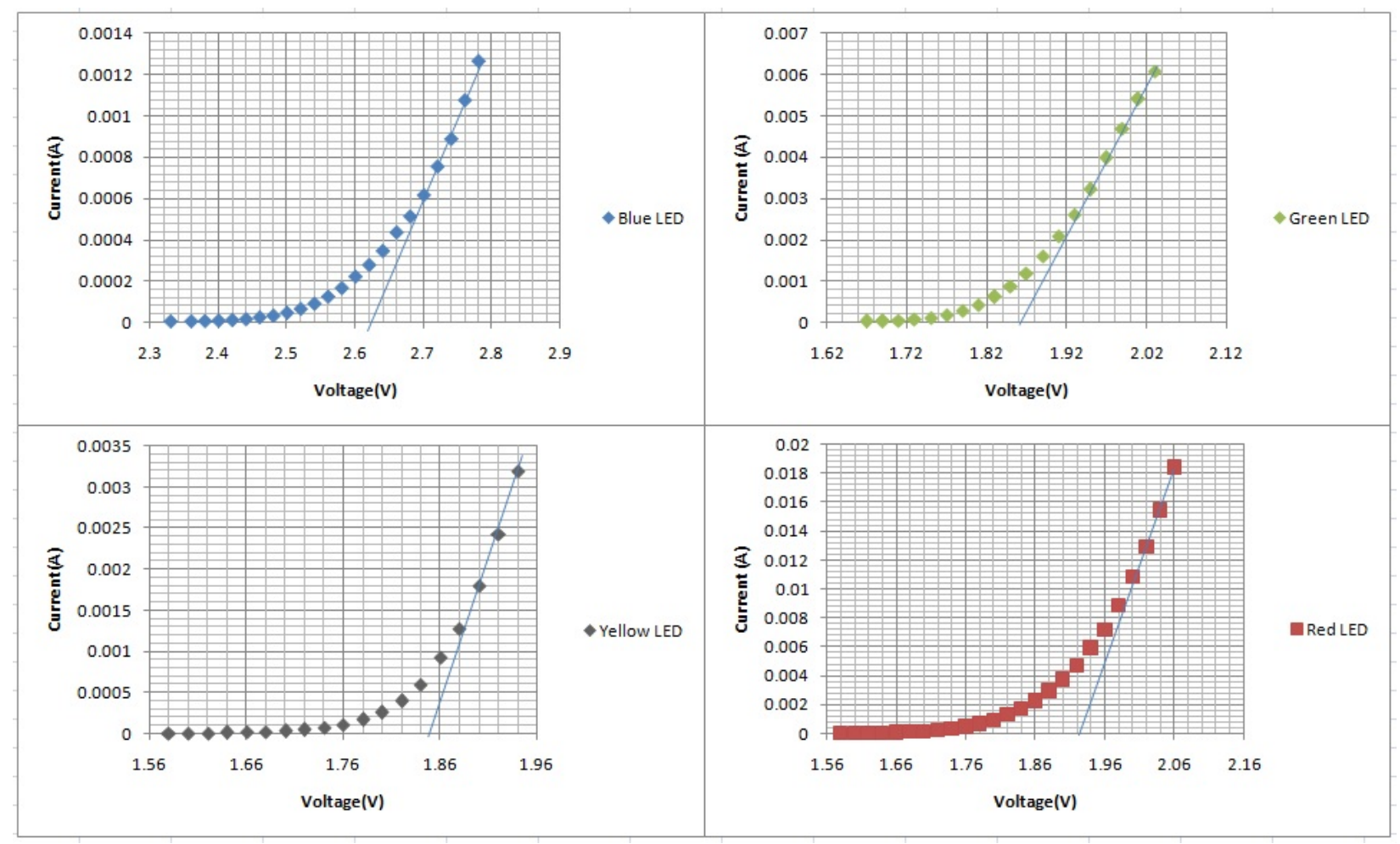

Figure 2. I-V-Characteristics of Blue, Green, Yellow and Red LEDs 


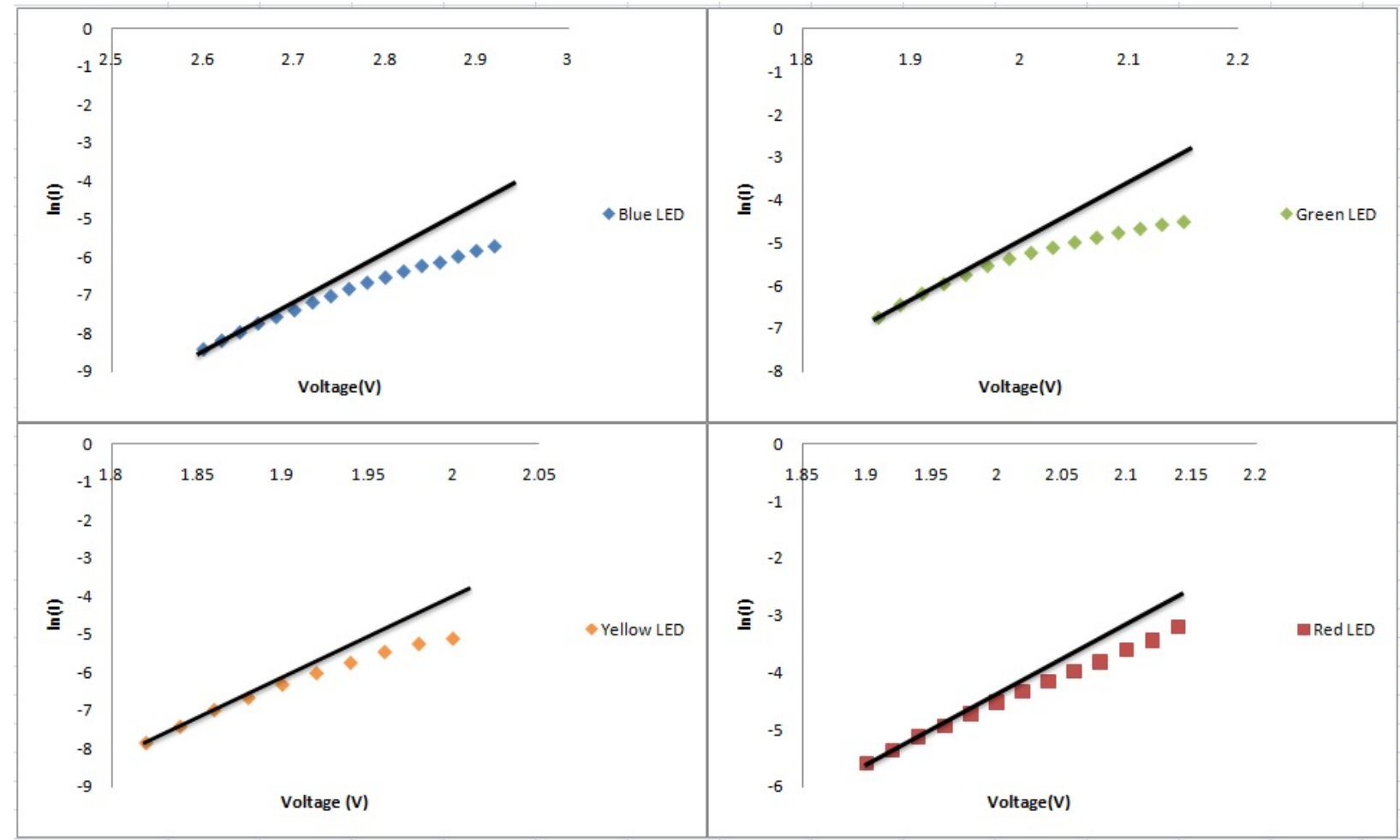

Figure 3. $\ln (\mathrm{I})$ vs. Voltage of Blue, Green, Yellow and Red LEDs

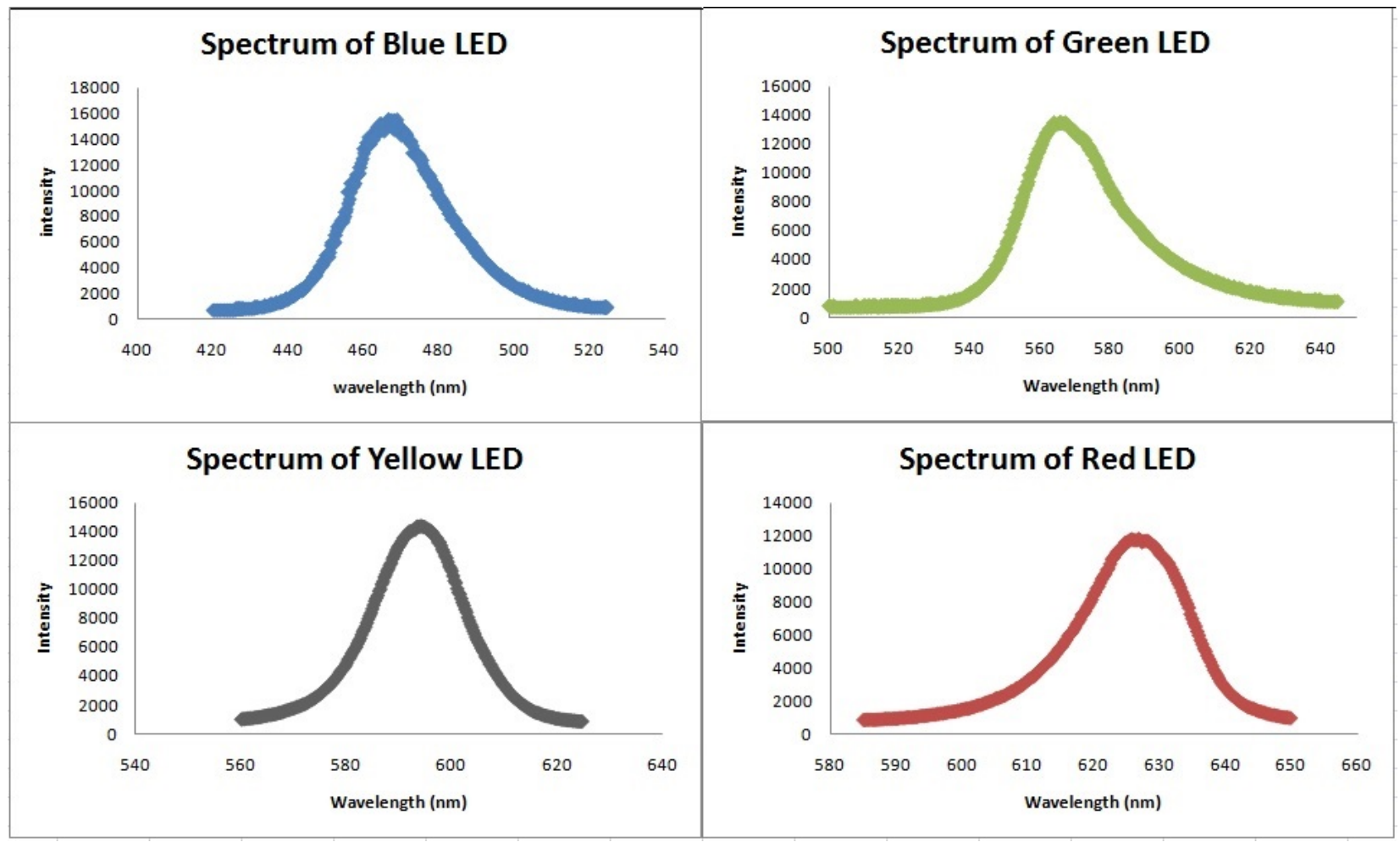

Figure 4. Spectrum of Blue, Green, Yellow and Red LEDs 